\title{
PROGRAMA DE SAÚDE NA ESCOLA: PERCEPÇÃO MULTIPROFISSIONAL NA PROMOÇÃO DA ALIMENTAÇÃO SAUDÁVEL EM UMA ESCOLA DO CABO DE SANTO AGOSTINHO-PERNAMBUCO
}

\author{
Cristiane Rodrigues de Carvalho ${ }^{1}$ \\ Cybelle Gomes de Souza ${ }^{2}$ \\ Cristiano do Nascimento Siqueira ${ }^{3}$
}

\begin{abstract}
A infância é uma fase que influencia diretamente, na adolescência, na vida adulta, bem como na senilidade. Sendo assim, hábitos alimentares incorretos nessa etapa podem desencadear uma série de problemas na vida futura dos indivíduos. A obesidade infantil é uma das consequências da má alimentação , e a determinação desta patologia é complexa e envolve, principalmente, fatores relacionados ao estilo de vida, como alimentação e atividade física, bem como condições socioeconômicas, culturais e demográficas, além dos fatores de ordem secundária, menos frequentes, como os genéticos e os distúrbios hormonais. Para o enfrentamento deste distúrbio, faz-se necessária a implementação de políticas públicas entre as quais aquelas voltadas ao ambiente escolar, capazes de exercer papel fundamental na promoção da saúde, da atividade física e da educação alimentar e nutricional. O Programa Saúde na Escola é uma das ações para a prevenção de problemas nutricionais e faz parte do eixo de Promoção da Saúde, presente no Plano de Ações Estratégicas para o Enfrentamento das Doenças Crônicas Não Transmissíveis no Brasil - 2011-2022. O Plano tem, como um de seus objetivos, reduzir a prevalência de obesidade em crianças, e é crucial para a manutenção da saúde alimentar de crianças e adolescentes.
\end{abstract}

Palavras- chave: Programa saúde na escola. Nutrição. Promoção da saúde. Educação alimentar. Educação em saúde.

\section{INTRODUÇÃO}

Com o advento da urbanização e da industrialização as situações de mánutrição aumentaram as taxas de morbimortalidade que são resultados das deficiências nutricionais e a ingestão excessiva de alimentos, que reflete no aumento do número de agravos crônicos não transmissíveis na sociedade.

A dieta é um fator importante para promover a existência, crescimento e desenvolvimento apropriado de uma criança (SANTOS, 2019).

Sendo necessário, respeitar as idades e fases alimentares dos indivíduos, para a manutenção da saúde. A introdução de alimentos complementares prematuramente, por exemplo, pode aumentar as taxas de 
morte na infância, devido à baixa ingestão dos elementos presentes no leite materno, que garantem a proteção da criança (SANTOS, 2019).

Monitorar a qualidade dos alimentos consumidos na fase infanto-juvenil é imprescindível, pela frequente prática de jejum, dietas irregulares e restritas, consumo compulsivo ou frequente de alimentos altamente energéticos, ricos em açúcares e gorduras, em substituição de alimentos adequados. Estas práticas não saudáveis, são responsáveis pela expressão da obesidade, sobrepeso, carências nutricionais e prevalência de doenças crônicas não transmissíveis (SILVA, 2015).

Nesse sentido, Políticas para a manutenção da saúde são primordiais, a Promoção da Alimentação Saudável (PAS), por exemplo, constitui um eixo integrador da Política nacional de Promoção da Saúde e foi implementada no Sistema Único de Saúde (SUS) em 2006.

Através de ações intersetoriais coletivas, individuais e ambientais que articulam estratégias para realização de práticas alimentares saudáveis e desenvolvimento ambiental sustentável, a PAS foi definida como uma das diretrizes da Política Nacional de Alimentação e Nutrição (PNAN) (BATISTA, 2017).

As principais metas da PAS são eliminação da má nutrição, erradicação da fome, redução das condições clínicas patológicas associadas ao excesso de peso além de promover a melhoria da qualidade de vida populacional.

O nível de complexidade dos problemas relacionados à alimentação condiciona o estado a redefinir suas práticas preventivas, sendo a unidade escolar o cenário ideal para desenvolvimento de tais ações.

Através das ações de abordagem integral da PAS consegue-se prevenir patologias relacionadas a carências nutricionais (desnutrição e avitaminoses), eleva-se a imunidade corporal tornando o indivíduo mais resistente contra doenças infecciosas, reduz as taxas de obesidade e sobrepeso, além de minimizar fatores de riscos para doenças crônicas não transmissíveis a exemplo da diabetes e hipertensão arterial (CASTILHO, 2018; BATISTA, 2017).

Para grande parte das crianças e adolescentes, a escola é um ambiente frequentado diariamente, esse espaço é de grande importância na promoção da saúde, pelo papel destacado na formação cidadã, no estímulo da autonomia, o exercício dos direitos e deveres, o controle das condições de saúde e qualidade 
de vida, bem como na obtenção de comportamentos e atitudes considerados como saudáveis (CAMOZZI,2015).

A promoção da alimentação saudável apresenta-se, neste sentido, como estratégia educacional de alta relevância no enfrentamento à obesidade de escolares infanto-juvenis. Esta estratégia é colocada em prática pela equipe multiprofissional (Médicos, Enfermeiros, Psicólogos e Nutricionistas) que assistem a unidade educacional através do Programa de Saúde na Escola (BATISTA, 2017).

\section{DESENVOLVIMENTO}

\section{Programa Saúde na Escola}

O Programa de Saúde da Família foi implantado em 05 de dezembro de 2007 através do Decreto Presidencial 6.286/07 como política Intersetorial brasileira integrando ações e estratégias do Ministério da Saúde (MS) e Ministério da Educação (MEC).

Objetivando ampliar o acesso de alunos da rede pública às ações preventivas em saúde, o PSE apresenta-se como uma nova política de Educação em Saúde.

Para o Ministério da Saúde, o PSE, tem como finalidade fornecer uma série de ações para prevenir, promover e atender á saúde de crianças, adolescentes e jovens do ensino básico público, com o fortalecimento e a sustentação da articulação entre as escolas públicas e as equipes da Estratégia Saúde da Família (ESF), através da realização de ações dirigidas aos alunos (MACHADO, 2015).

O PSE objetiva-se na estruturação e no oferecimento de serviços em uma área geográfica (território) e na implementação de uma rede de corresponsabilidades para assegurar a sustentabilidade das ações. Os gestores do PSE estão ordenados, no âmbito nacional, pela Comissão Intersetorial de Educação e Saúde na Escola (CIESE), pelas equipes do Ministério da Educação (MEC) e do Ministério da Saúde (MS) que compõem o Grupo de Trabalho Intersetorial Federal (GTI-F) (MARTINS, 2020). 
A escola é um equipamento importante para estruturação de uma sociedade democrática. Logo, é um espaço potencial de transformações sociais e de constituição de conhecimentos e valores e de implementação de políticas públicas que visem à redução das chamadas vulnerabilidades da ordem individual, social e institucional, que prejudicam o crescimento e desenvolvimento pleno do público juvenil (BRASIL, 2015).

O Programa é executado com base em cinco componentes: Avaliação das condições de saúde das crianças, adolescentes e jovens das escolas públicas; Promoção da saúde e ações de prevenção de doenças e de agravos à saúde; Educação continuada e capacitação dos profissionais da educação e da saúde e de jovens; Monitoramento e avaliação da saúde dos estudantes; Monitoramento e avaliação do programa (CAVALCANTI; LUCENA; LUCENA, 2015).

O benefício na adesão ao programa é demonstrado através dos dados divulgados pela Pesquisa Nacional de Saúde do Escolar (PeNSE) que em 2015 declarou que os estudantes das escolas assistidas pelo PSE apresentaram uma redução no consumo de guloseimas, refrigerantes e substâncias psicoativas.

Ainda de acordo com esta pesquisa a obesidade foi diagnosticada em $7,8 \%$ dos adolescentes entre 13 e 17 anos, atingindo mais o sexo masculino $(8,3 \%)$ do que o feminino $(7,3 \%)$.

Nas crianças entre 5 e 9 anos, indicou um aumento na prevalência de sobrepeso de 34,8\% e 16,6\% no índice de obesidade, gerando uma situação de alerta para as equipes do PSE responsáveis pela implantação da Promoção da Alimentação Saudável (PAS) no contexto Escolar.

Em Pernambuco o índice de obesidade também aumentou de 12,3\% para $17 \%$ colocando o Estado no sexto lugar do ranking nacional. O estado pactuou 4872 escolas e obteve adesão ao PSE em todos os seus 185 municípios. São 2040 equipes de PSE atuando nas escolas e atingindo com suas ações mais de 1 milhão de alunos (1.156.289 escolares) de acordo com os Dados do Painel de Adesões ao PSE-2017. Práticas alimentares incorretas, ausência de atividade física, surgem como fatores condicionantes desta realidade, justificando mais uma vez a importância do PAS através do PSE no contexto escolar. 
A intersetorialidade tem sido considerada como uma iniciativa de oposição à desagregação de políticas sociais, indispensável para superar as iniquidades bem como, para a manutenção da saúde e da qualidade de vida da sociedade, principalmente nos países de baixa e média renda per capita. Os debates a cerca dos significados e das atribuições desata palavra são amplos, e ao mesmo tempo, genéricas. discussões em torno dos seus significados e atribuições são vastas e, ao mesmo tempo, genéricas. Em meio aos vários significados de intersetorialidade há confluência de que pode ser entendida como a articulação entre diferentes áreas e setores, o compartilhamento de poderes, ideias e saberes, com o intuito de atuar de forma integrada a respeito dos impasses e demandas em busca da melhoria da qualidade vida (SOUSA; ESPERIDIÃO; MEDINA, 2017).

No âmbito da saúde a intersetorialidade é uma questão-chave para a promoção da saúde, já que a complexidade das questões sociais encontradas na escola torna pequena ou nula a possibilidade de apenas um setor conseguir ser efetivo em sua resolução ou atenuação (CARVALHO, 2015).

Políticas de caráter intersetorial precisam de articulações e arranjos por meio de parcerias entre diferentes setores e segmentos sociais como: educação, saúde, cultura, esporte, lazer, empresas privadas, organizações não governamentais (ONG), fundações, entidades religiosas, as três esferas de governo e organizações comunitárias ( SA, 2019).

As políticas de saúde no contexto brasileiro, já têm se desenvolvido no reconhecimento da intersetorialidade. Nunca antes na história, proferiu-se tanto em relação á saúde e a promoção da mesma como na atualidade, o que colabora, para o reconhecimento do papel de promoção da saúde vinculada ao âmbito escolar. Neste interim, identifica-se a necessidade de atuação não apenas no setor saúde como também no resultado de ações articuladas e multidisciplinares, tornando a intersetorialidade uma condição para á prática da Promoção da Saúde (FARIAS, 2016).

A articulação entre Escola e Rede Básica de Saúde é a base do Programa Saúde na Escola. O PSE é uma política de saúde na escola que se projeta a 
integrar permanentemente a saúde e educação com ações que buscam melhorar a qualidade de vida dos alunos de escolas de educação básica, tendo por base ações de prevenção, promoção e atenção à saúde. Objetiva-se em propor uma prática educativa emancipatória, que transforma saberes existentes, no intuito de fomentar o desenvolvimento da autonomia e da responsabilidade dos indivíduos no cuidado com a saúde (SA, 2020).

As condutas de saúde na escola (ou higiene escolar) receberam notabilidade a partir do ano de 1889, com medidas sanitaristas, em vista das precárias condições de vida da população, consequentes da falta de um sistema de saúde pública e presença de epidemias. Estas foram elaboradas com o intuito do decrescimento da incidência de enfermidades. Apenas na década de 1950, os programas foram direcionados á saúde na escola com ações voltadas às questões biológicas das problemáticas relacionadas à educação (COUTO et al., 2016).

Lamentavelmente a ligação entre os serviços de saúde e as escolas é considerado frágil, com isso, observa-se cada vez mais, a necessidade entre a articulação entre a saúde e a educação para que haja o sucesso nas implementações de ações voltada para a saúde dos estudantes. (FARIAS, 2016).

Em frente a este cenário, nota-se a relevância de se redefinirem as relações entre profissionais da saúde, instituições de ensino e comunidade, e se reorientarem os processos formativos para atuação no setor saúde, de maneira a implementar $\mathrm{o}$ atendimento integralmente e humanizado á sociedade (SILVA, 2015).

Avalia-se, ainda, a importância de maior integração ensino-serviçocomunidade, de forma a transformar os cenários de aprendizagem e desenvolvimento, e superar as concepções tradicionais e bancárias de educação (SILVA, 2015).

O Programa Saúde na Escola resulta diretamente nesta necessidade de articulação justamente para ampliar o alcance e o impacto das ações de saúde aos estudantes e seus familiares, por meio de ações intersetoriais. No PSE, a 
proposta é que essa articulação ocorra, principalmente, entre as Equipes de Saúde da Família (ESF) e as escolas do território de adstrição dessas equipes, para que haja o atendimento necessário da população (FARIAS, 2016).

\section{Programa Saúde na Escola e Alimentação}

Atualmente, $55,7 \%$ da população adulta do Brasil se encontra com sobrepeso e 19,8\% está obesa, de acordo com a Pesquisa de Vigilância de Fatores de Risco e Proteção para Doenças crônicas por Inquérito Telefônico (Vigitel), de 2018. O Ministério da Saúde alerta que é imprescindível a adoção de hábitos saudáveis para a diminuição dos casos de excesso de peso e das doenças que advém da obesidade (BRASIL, 2020).

Dados do Vigitel mostram ainda que $7,7 \%$ da população adulta apresenta diabetes e $24,7 \%$, hipertensão - doenças que podem estar relacionadas à obesidade. A Pesquisa Nacional de Saúde (PNS), de 2013, indica que, dentre os adultos com diabetes, $75,2 \%$ têm excesso de peso e, entre os adultos com hipertensão, 74,4\% têm excesso de peso. Sendo assim, é importante a melhoria dos hábitos para a manutenção do peso e prevenção de doenças (BRASIL, 2020).

A obesidade é uma condição que atinge não apenas adultos, como os mais novos. Durante a adolescência, dá-se a modificação e consolidação de diversos hábitos. Nessa idade, passa-se por importantes mudanças biológicas, cognitivas, emocionais e sociais, experimentam-se novos comportamentos e vivências, sendo algumas dessas experiências fatores de risco para a saúde, como, consumo de álcool, alimentação inadequada e sedentarismo, entre outros (OLIVEIRA,2017).

Esses fatores podem dar início a uma série de doenças crônicas não transmissíveis (DCNT), que podem ser diabetes, câncer, doenças cardiovasculares, principais causas de morte em adultos no país adultos no país e no mundo.

Estas doenças e a obesidade doenças relacionadas à alimentação são desafios globais e o seu controle e prevenção exigem a implementação de políticas intersetoriais (BRASIL, 2020). O Programa Saúde na Escola, é uma das iniciativas que promovem a saúde e a educação de maneira integral, articulando saúde e escola. Atualmente, o programa está presente em 91.659 escolas de 
5.289 municípios. Em 2019, foram realizadas 53.040 ações de atividade física e 240.139 de alimentação saudável e prevenção da obesidade (BRASIL, 2020).

O PSE faz parte do eixo de Promoção da Saúde, presente no Plano de Ações Estratégicas para o Enfrentamento das Doenças Crônicas Não Transmissíveis (DCNT) no Brasil - 2011-2022. Este plano, lançado em 2007 pelos Ministério da Saúde e Ministério da Educação tem como finalidade, contribuir para a formação integral dos estudantes da rede pública de ensino, por meio de ações integradas e articuladas entre as escolas e as Equipes de Saúde da Família (ESF).

Segundo as normas do Programa, o município deve realizar, minimamente, algumas ações obrigatórias, como a avaliação antropométrica dos escolares e a promoção da alimentação saudável e prevenção da obesidade infantil (BATISTA, 2019).

\section{Desnutrição}

A desnutrição é a condição de baixa reserva calórico-proteica, em que o organismo pode apresentar casos leves, moderados ou graves de evolução normal de seus parâmetros bioquímicos, funcionais e anatômicos (VIANA, 2018).

Possui etiologia complexa, estando ligada às condições socioeconômicas ambientais, maternas, relacionadas às práticas alimentares infantis, morbidades e de acesso aos serviços de saúde (ARAÚJO, 2016).

Muitos estudos a nível mundial também apontam que a desnutrição infantil está relacionada ao baixo rendimento familiar, ao elevado número de filhos, iliteracia parental, falta de alimentação em exclusivo ao seio materno até aos 6 meses de vida e práticas errôneas de desmame e alimentação (PRETO,2018).

A desnutrição é apontada como uma doença e pode causar malefícios e efeitos irreparáveis ao longo do desenvolvimento físico e mental da criança, resultando em danos de memória e concentração, perda de peso, retardamento no desenvolvimento psicomotor, dificuldade de aprendizagem, impulsionando-a a comportamentos agressivos e negativistas entre outros. Estes aspectos 
favorecem a diminuição da imunidade destas crianças e aumentam as chances de contaminação, tornando-as mais suscetíveis a doenças (MENDES, 2016).

Em vista da infância ser um período de elevado desenvolvimento físico, psicológico e social, para um crescimento saudável é preciso que seja disponibilizada a esta criança recursos como; uma alimentação correta, afeto e educação (FELBERG; PINHEIRO; BATISTA, 2018).

Isso também, por que a subnutrição representa um problema relevante na saúde pública em países de baixa e média renda, em razão da sua grande magnitude e impacto sobre a morbimortalidade infantil, com prevalências que variam de 43 a 59\% sendo necessária melhoria neste setor (ARAÚJO, 2016).

\section{Nutrição}

A Segurança Alimentar e Nutricional (SAN) compreende a realização do direito de todos ao acesso regular e permanente a alimentos de qualidade, em quantidade suficiente, sem comprometer 0 acesso a outras necessidades essenciais, tendo como base práticas alimentares promotoras de saúde que respeitem a diversidade cultural e que seja integralmente sustentável. (BOARETTO, A.\& NATALE, 2016). A realização destas boas práticas de alimentação e nutrição atuam como requisitos básicos para a promoção e a proteção da saúde, possibilitando a afirmação plena do potencial de crescimento e desenvolvimento humano, com qualidade de vida e cidadania, sendo sua concretização responsabilidade tanto do Estado, quanto da sociedade e dos indivíduos (CAVALCANTI, 2017).

Entretanto, dificilmente são integralmente executadas as práticas alimentares nutricionalmente corretas, visto que, uma a cada três pessoas no mundo, possuem alguma forma de carência nutricional, seja por deficiência de vitamina $A$, carência de ferro ou iodo e mais de meio bilhão de pessoas a nível mundial apresentam manifestações clínicas dessas alterações nutricionais, como baixa no sistema imunológico, cegueira, anemia e retardo mental (DE SOUZA, 2015).

Além das carências nutricionais, o excesso de peso, representa um problema de saúde pública. Dados obtidos por meio de uma pesquisa, aponta que $52,5 \%$ dos brasileiros encontram-se acima do peso, o que é um fator de 
risco para doenças crônicas que são causas de $72 \%$ de óbitos do Brasil. Dentro dessa porcentagem, encontra-se em grande proporção indivíduos com idade superior a 18 anos (BRASIL, 2015).

Estes impasses relacionados a saúde nutricional, é o resultado de diversas razões. Fatores, psicológicos, emocionais, sociais, religião, e todos os outros fatores ambientais e internos, podem influenciar nos hábitos alimentares pois estes, definem o comportamento alimentar que está relacionado as reações frente ao alimento, envolvendo todas as ações relacionadas ao ato de se alimentar (com quem comer, onde comer, o que comer e como comer) (ALVARENGA ; FIGUEIREDO ; TIMERMAN ; ANTONACCIO, 2015).

Além do ambiente que o indivíduo está inserido, ambientes de trabalho com condições desfavoráveis associadas a longas jornadas de trabalho, por exemplo, exercem influência no aparecimento de obesidade (FREITAS; ASSUNÇÃO; BASSI; LOPES, 2016).

Distúrbios no comportamento alimentar provenientes de alterações psicológicas como ansiedade, estresse e atitudes alimentares a fim de reduzir tensões, também pode estar relacionado a dificuldade na manutenção de hábitos saudáveis visto que, podem ocasionar o consumo excessivo de alimentos resultando em obesidade (FERREIRA, 2018).

Estes distúrbios relacionados à insatisfação corporal e consequentemente às mudanças no comportamento alimentar ocorrem na adolescência, mas predomina também na fase adulta devido à preocupação na redução do peso associada ao padrão de magreza. A insatisfação corporal tem maior prevalência no sexo feminino e possui associação com o estado nutricional, pois quanto maior o peso e valor de IMC, maior a insatisfação corporal (POLL; ASSMANN; MOLZ; 2016; TORAL; GUBERT; SPANIOL; MONTEIRO, 2016).

Os impasses relacionados a alimentação são constatados em todas as fases de vida dos indivíduos. Na fase da infância, a obesidade é uma das enfermidades manifestadas. Esta doença tem aumentado consideravelmente em níveis mundiais tornando-se uma epidemia preocupante nos últimos anos. Devido aos grandes índices de casos, vários estudos estão sendo desenvolvidos, muitos deles, focados na complexa gravidade da doença (LINHARES, 2016). 
Algumas das causas da obesidade na infância estão relacionadas com o sedentarismo. Atualmente, a maioria das atividades de lazer das crianças não envolve exercício físico, pois passam muito pelo computador, a televisão e os jogos de consolas. Esta inatividade aumenta a potencialidade das crianças virem a ganhar peso (PEREIRA; LOPES, 2016).

Outros determinantes etiológicos da obesidade infantil é a ingestão de alimentos industrializados e a exposição excessiva da criança à televisão. Isso por que, o meio televisivo, é um portal de acesso a diversos meios de propaganda, inclusive de alimentos industrializados e de baixo valor nutricional, que são ofertados em grande quantidade e estimulam a ingestão e a aquisição, e diminui o consumo e o desejo por alimentos com o valor nutricional adequado (COSTA, 2017).

Dessa forma tornam-se fundamental, ações que objetivam o despertar para a importância da alimentação e nutrição infantil, com capacitação dos profissionais para o uso do fortificante alimentar, bem como para o conhecimento dos benefícios da alimentação adequada na infância, e os malefícios decorrentes da falta da mesma (DE SOUZA, 2015).

\section{As influências alimentares}

As preferências e aversões alimentares são estabelecidas nos primeiros anos e prosseguem até a fase adulta. Dessa forma, as principais influências na ingestão alimentar nos anos de desenvolvimento incluem o ambiente familiar, tendências sociais, a mídia, pressão dos colegas e doença (ANTANA; OLIVEIRA, 2015).

Os pais possuem importante papel na formação do hábito alimentar infantil. As escolhas alimentares parentais, em relação à quantidade e qualidade dos alimentos podem determinar o comportamento alimentar das crianças (MELO, 2017).

O âmbito familiar e social é indicador de vasta indução e influência no aparecimento de obesidade infantil. Outra causa de grande contribuição para a obesidade em crianças é a presença de obesidade na família, mais precisamente 
nos pais. Além de fatores genéticos envolvidos, a obesidade infantil, pode estar relacionada questões de aprendizagem de hábitos alimentares (LINHARES, 2016).

Os meios de comunicação também influenciam o consumo dos alimentos, pois a alimentação envolve tanto o desejo quanto a necessidade da criança. A quantidade de produtos destinados à criança tem seu apelo infantil exagerado, sendo uma estratégia utilizada pela mídia para lucrar mais com o público infantil (DE CARVALHO, 2016).

A televisão é um dos meios de comunicação mais acessados para o entretenimento e educação, e tem um papel crucial na socialização, além de ser um espaço de grande influência para a aquisição de produtos, preenchendo o universo das crianças com imagens irreais (DE CARVALHO, 2016).

As estratégias de marketing buscam seduzir para o consumismo alimentar, utilizando-se de artifícios emocionais e afetivos, bem como da oferta de brindes e uso de personagens e apresentadores infantis a fim de atrair a atenção das crianças para o consumo de seus produtos (PIMENTA; ROCHA; MARCONDES, 2015).

A indústria alimentar vê a criança como sujeito consumidor e está cada vez mais atenta ao fato de elas terem grande influência na decisão de compra dos pais, devido ao seu considerável poder de importunação (CECCATTO, 2018).

Existem defensores da utilização da televisão pela visão desta, com um meio de comunicação e como um meio de aprendizado, e da intensificação de capacidades perceptomotoras, enquanto outros criticam, por acreditarem que 0 meio televisivo, desestimula a leitura, a brincadeira e com isso tem mais facilidade de apresentar a obesidade, além de diminuir a interação social e de estar vinculada ao consumismo na infância e por causar dependência (DE CARVALHO, 2016) .

\section{Cantina escolar: Efeitos na alimentação infantil}


Estudos acerca dos alimentos consumidos no ambiente escolar indicam um elevado consumo de alimentos com escasso valor nutricional e de alta densidade energética, independentemente da forma de aquisição, seja por cantinas ou alimentos levados de casa para a ingestão na escola (ROSSI, 2019).

A cantina escolar é conceituada com um espaço dentro de ambientes de ensino que tem como objetivo fornecer alimento, a alunos, professores e demais indivíduos que frequentem o ambiente, com o recebimento de pagamento. Outrossim, cabe salientar, que grande parte das cantinas escolares ofertam alimentos com baixo valor nutricional e altos teores de gordura, açúcar e sódio, alimentos estes, estimados entre os escolares (PRADO, 2018).

Neste sentido, em agosto de 2008, foi promulgada a Lei $n . .013 .027$, que dispõe sobre a comercialização de lanches e bebidas em escolas no âmbito do Estado do Rio Grande do Sul, na qual afirma que o comércio destas cantinas escolares devem fornecer preferencialmente produtos e serviços que tenham como objetivo garantir a saúde dos alunos (GIACOMELLI, 2017).

Estabelece, além disso, que as cantinas forneçam alimentos como: frutas, sanduíches, sucos e saladas naturais em maior quantidade que os demais alimentos. Proíbe a exibição de cartazes com propagandas publicitárias que estimulam o consumo de balas, chicletes, salgadinhos e refrigerantes. Exige a fixação, em local visível, de painéis que tratem de assuntos a respeito de qualidade nutricional dos alimentos e o alvará sanitário expedido pelo órgão competente. Declara ainda às qualidades higiênico-sanitária e nutricional dos alimentos vendidos, e o dever dos donos de cantinas de cumpri-las (GIACOMELLI, 2017).

Embora haja a regulamentação das ofertas de alimentos nas cantinas, há estudos que apontam que os alimentos proibidos são comercializados normalmente, isso por que não existe uma fiscalização adequada para que o regulamento seja cumprido. Observa-se que apenas a regulamentação não é suficiente para a mudança de hábitos alimentares, esta mudança deve ser construída desde os primeiros anos de vida no âmbito familiar e a escola é ambiente favorável para a inserção de hábitos alimentares saudáveis (PRADO, 2018). 
A Organização Mundial da Saúde (OMS) e pelo Ministério da Saúde, indicam através do manual das cantinas escolares saudáveis, a implementação de medidas para a melhoria da alimentação infantil, como a promoção de práticas alimentares saudáveis nas escolas, bem como a regulamentação do comércio de alimentos nas cantinas escolares (GIACOMELLI, 2017).

A oferta de alimentos com baixo valor nutricional, e a falta de percepção dos alunos a respeito da alimentação saudável, são causadoras de efeitos maléficos para saúde infantil, sendo necessária a intervenção de autoridades e de mais políticas públicas para a garantia da saúde no ambiente estudantil (SANTOS, 2018).

\section{Sedentarismo Infantil}

O sedentarismo está relacionado à baixa prática de atividade física ou a falta de práticas esportivas que levam o sistema biológico e cognitivo, acomodarem-se nas facilidades que a vida moderna proporciona. Isto inclui principalmente tecnologias, as quais são ferramentas fortes em transformar de forma ágil os bons hábitos alimentares em dependência, ocasionando graves riscos de saúde, uma vez que a utilização das tecnologias digitais está presente em todas as situações da vida (TASCA, 2018).

Quando se ingere uma quantidade de calorias acima do que é necessário e que não seja proporcional ao gasto que teve durante um tempo podem-se desenvolver patologias. $O$ individuo que não controla o consumo de calorias juntamente com o gasto, pode gerar um acumulo de calorias e com isso se tornar obeso (REIS, 2017).

No Brasil, apenas $34,4 \%$ dos adolescentes acumulam 300 minutos ou mais de atividades físicas por semana, com percentual menor entre meninas (25,4\%). Em adição, 60\% dos adolescentes assistem a TV mais de duas horas por dia em um dia de semana (IBGE, 2016).

Isso contribui, para o desenvolvimento da obesidade e de outras doenças crônicas não transmissíveis. 
No ambiente escolar a presença de exercícios físicos pode se tornar ótimas atividades, de grande importância, pois proporciona o desenvolvimento de um ambiente prazeroso e motivador, além de contribuir para a qualidade de vida e promoção da saúde dessas crianças (REIS, 2017).

A Organização Mundial de Saúde, indica que a prática regular de atividade física, previne as doenças crônicas não transmissíveis. Quando se refere em especial as crianças e os adolescentes, as atividades físicas interagem com a dieta saudável de maneira benéfica, pois diminui a utilização do álcool, de drogas, do tabagismo, aumenta a interação social e diminui a violência (MOLINA; CADE, FARIAS JÚNIOR, 2018).

\section{Educação alimentar e nutricional}

Com o passar dos anos, as sociedades alcançam novos hábitos alimentares, normas, aos quais incorporam um processo de experimentação social que, por tentativa e erro, propicia o desenvolvimento de um saber nutricional. Com isso, considerar que os hábitos alimentares da população necessitam ser mudados é um grande desafio para as políticas públicas e para a educação em nutrição, visto que esses compreendem relações entre pessoas e comportamentos humanos (CERVATO-MANCUSO; VINCHA; SANTIAGO, 2016).

Os níveis de excesso de peso e consequentemente de doenças crônicas, por exemplo, estão em crescente avanço, é o que aponta Pesquisa de Vigilância de Fatores de Risco e Proteção para Doenças crônicas por Inquérito Telefônico (Vigitel), de 2018, do Ministério da Saúde. Sobre esse índice, houve aumento de $67,8 \%$ nos últimos treze anos, saindo de $11,8 \%$ em 2006 para $19,8 \%$ em 2018 (BRASIL, 2019).

O Vigitel também demonstrou que o excesso de peso entre a população brasileira teve um crescimento considerável. No Brasil, mais da metade da população, 55,7\% tem excesso de peso. Um aumento de 30,8\% quando comparado com percentual de 42,6\% no ano de 2006 (BRASIL, 2019).

Frente a este cenário, o excesso de peso tornou-se prioridade para 0 campo das políticas públicas no país, especialmente para o campo da 
alimentação e nutrição. Nesta direção, a proposição de estratégias e iniciativas que permitam a prevenção e o controle do excesso de peso são extremamente importantes dentre as que envolvam atividades de Educação Alimentar e Nutricional (EAN) (ARAÚJO, 2017).

Constata-se, a partir de então, como a EAN se torna conveniente no que se diz às políticas públicas de alimentação e nutrição devido à necessidade de ampliar a discussão sobre as possibilidades, limites e o modo como a EAN é realizada, resultando em ações governamentais, especialmente as desenvolvidas pelos Ministérios do Desenvolvimento Social e de Combate à Fome, levando à construção do Marco de Referência de Educação Alimentar e Nutricional para as Políticas Públicas, que reflete uma importante etapa de valorização dessas ações. (GREENWOOD; FONSECA,2016)

A Educação Alimentar e Nutricional (EAN) é um campo de ação da Segurança Alimentar e Nutricional (SAN) e da promoção da saúde, e é tida como uma estratégia crucial para prevenir e controlar problemas alimentares e nutricionais contemporâneos, como as doenças crônicas não transmissíveis , dentre elas : doenças cardiovasculares, diabetes mellitus, doenças respiratórias crônicas.

A EAN é uma ferramenta importante a ser adotada na prática clínica para auxiliar na minoração do peso corporal e dos fatores de risco associados ao excesso de peso, na adesão ao tratamento da obesidade e na mudança de estilo de vida, com repercussões positivas em longo prazo. (DE ALMEIDA; PEREIRA, 2015). A EAN contribui, ainda, para a valorização das diferentes expressões da cultura alimentar, o fortalecimento de hábitos regionais, a redução do desperdício de alimentos e a promoção do consumo sustentável e da alimentação saudável (BORSOI; TEO; MUSSIO, 2016).

Os mecanismos de ensino-aprendizagem empregados nas ações do programa necessitam se diferenciar dependendo do ambiente que for realizado e o público-alvo, e para a escolha dos métodos e temas, é crucial avaliações através de entrevistas ou de questionários. A utilização de atividades lúdicas, principalmente com escolares, tem-se mostrado efetiva na ampliação do conhecimento sobre alimentos e nutrição, pois estimulam a compreensão do 
conteúdo abordado de forma fácil e prazerosa e refletem a realidade vivenciada em sua faixa etária (PRADO, 2016).

O ambiente escolar é considerado um cenário oportuno não somente por promover ações educativas bem como para ampliar o acesso a uma alimentação saudável através de estratégias de intervenções nutricionais. (ARAÚJO, 2017).

É neste contexto, considerando o papel da escola na direção de viabilizar qualificar a inserção e a ação do estudante no mundo, que a escola tem sido um dos espaços mais focados pelas políticas públicas de alimentação e nutrição no Brasil, conferindo, mais recentemente, especial ênfase ao desenvolvimento de educação alimentar e nutricional neste espaço ( BORSOI ; TEO; MUSSIO, 2016).

$\mathrm{Na}$ comunidade escolar, diretores, coordenadores, professores, merendeiras, donos e funcionários de cantinas escolares são profissionais que devem ser incentivados a envolver-se nessas ações, para diversificar os métodos educativos e aproximar o saber técnico e popular (PRADO, 2016).

\section{Pesquisa qualitativa: programa saúde na escola}

\section{Área de estudo}

O município do Cabo de Santo Agostinho está inserido na Mesorregião Metropolitana do Recife, distante $33 \mathrm{~km}$ da capital e localizado na Mata Sul do Estado. Possui uma população de 185.123 habitantes e elevada taxa de obesidade infantil. Pactuou suas 53 escolas municipais ao PSE. São 34 equipes atuando nas escolas e sensibilizando 12.479 alunos de acordo com os Dados do Painel de Adesões ao PSE-2017.

O estudo foi realizado com a equipe de PSE que assiste aos estudantes das Escolas localizadas no bairro da Charneca, no Cabo de Santo Agostinho. A base do programa fica situada na Escola Municipal Vereador João Cinaco da Silva, localizada à Rua 41 Loteamento Nilton Carneiro, Charneca, Cabo de Santo Agostinho-PE. A escola possui 195 alunos nos anos iniciais do ensino fundamental ( $1^{\circ}$ ao $5^{\circ}$ ano) e 170 alunos nos anos finais do EF (6을 ao $9^{\circ}$ ano). A unidade apresenta condições estruturais estáveis para favorecer o aprendizado dos escolares, porém não possui quadra de esportes que poderia ser um fator complementar a adesão ao Programa 
de Alimentação Saudável. Possui saneamento básico e abastecimento regular de água.

\section{Tipo de Pesquisa}

Pesquisa exploratória-descritiva de abordagem qualitativa que pretende identificar os obstáculos à efetivação da promoção da alimentação saudável no contexto escolar a partir da percepção dos Profissionais integrantes do Programa de Saúde na Escola. Este método permite observar, descrever e explorar o contexto escolar no qual a equipe de PSE está inserida.

A avaliação qualitativa permite conhecer a percepção destes profissionais acerca dos obstáculos impeditivos a plena efetivação do Programa de alimentação saudável nas unidades que assiste.

O estudo foi realizado com os integrantes da equipe do PSE (Médico, Enfermeiro, nutricionista, psicólogo, fonoaudiólogo) que atende as escolas municipais localizadas no bairro da Charneca no Cabo de Santo Agostinho e tem como base a Escola Municipal Vereador João Siríaco da Silva.

Como critério de elegibilidade fez-se necessário ser maior de 18 anos, lotado na equipe do Programa de Saúde na Escola que assiste as unidades educacionais da Charneca, bem como aceitar de forma espontânea participar da pesquisa. Foram excluídos do estudo aqueles participantes que estejam no programa em caráter substitutivo, de licença médica ou férias.

A coleta de dados aconteceu através de uma entrevista gravada e guiada por um roteiro semiestruturado. Aconteceu no entre os meses de outubro e novembro de 2018.

Com o propósito de avaliar a percepção da equipe multiprofissional do PSE acerca dos obstáculos à efetivação da PAS no contexto escolar, foram realizados os seguintes questionamentos:

1. Quais as estratégias para promoção da alimentação saudável são aplicadas nas unidades educacionais que você assiste?

2. Como acontece a interação entre gestão educacional e equipe multiprofissional de saúde?

3. Como você avalia o padrão alimentar e a vulnerabilidade a agravos decorrentes da má alimentação nos escolares que você assiste? Existem muitos casos de sobrepeso ou obesidade? 
4. Na sua visão, o que impede que as ações do PAS tenham um resultado efetivo?

5. Como você acha que estes obstáculos podem ser superados?

Os entrevistados responderam que práticas como: "palestras com nutricionistas sobre meios corretos e saudáveis de alimentação são oferecidos sempre dentro das ambiências escolares. Os profissionais envolvidos na pesquisa também alegaram que a relação junto aos gestores pode ser considerada positiva. Eles entendem que Educação e Saúde devem andar juntas. Além do mais os participantes afirmam que há uma grande incidência de jovens obesos ou com sobrepeso dentro das escolas. $E$ todos acham que de fato e de verdade as escolas devem abraçar o programa e vivenciar dentro das aulas e todos os dias.

\section{CONCLUSÃO}

O programa saúde na escola, em seu escopo, aborda pontos importantes para a prevenção e combate do excesso de peso infantil.

Combater e controlar este excesso de peso infantil requer esforços de diversas áreas e setores. A escola, ao propiciar experiências e trocas de saberes, mostra-se um ambiente potencialmente favorável à formação de hábitos de vida saudáveis, não só para as crianças e adolescentes como também para seus familiares e comunidade.

O excesso de peso nas escolas estudadas alcança cerca de uma a cada três crianças. A avaliação do estado nutricional e o desenvolvimento de temas relacionados a alimentação saudável e nutrição, além da promoção da prática de atividade física, estiveram presentes nos projetos político-pedagógicos dessas escolas.

Há necessidade de buscar maior uniformização do cardápio escolar das diferentes refeições quanto ao oferecimento de alimentos saudáveis. A presença de alimentos ultra processados no desjejum e no lanche da tarde é bastante expressiva, diferentemente do almoço, cuja participação de alimentos in natura e minimamente processados é satisfatória. 
Programas e políticas públicas focados no ambiente escolar devem ser constantemente avaliados, monitorados e, quando necessário, modificados, sempre considerando a participação dos diversos atores envolvidos, de modo que as ações a serem realizadas sejam pensadas e construídas coletivamente.

\section{REFERÊNCIAS}

ALVARENGA, M., Figueiredo, M., Timerman, F., \& Antonaccio, C. M. A (2015). Nutrição Comportamental. São Paulo: Manole.

ARAÚJO, Aillen Leite et al. O impacto da educação alimentar e nutricional na prevenção do excesso de peso em escolares: uma revisão bibliográfica. RBONE-Revista Brasileira de Obesidade, Nutrição e Emagrecimento, v. 11, n. 62, p. 94-105, 2017.

ARAÚJO, Thiago Santos de et al. Desnutrição infantil em um dos municípios de maior risco nutricional do Brasil: estudo de base populacional na Amazônia Ocidental Brasileira. Revista Brasileira de Epidemiologia, v. 19, p. 554-566, 2016.

BATISTA, Mariangela da Silva Alves. Proposta de plano de ação, no âmbito do Programa Saúde na Escola, para prevenção e controle da obesidade infantil em um Município da Grande São Paulo-SP. Bol Inst Saúde [Internet], p. 52-8, 2019.

BOARETTO, A.; NATALE, William. Importância da Nutrição Adequada para Produtividade e Qualidade dos Alimentos. Nutrição e Adubação de Hortaliças. Editora Prado, RM \& Cecílio Filho A. B, p. 45-74, 2016.

BORSOI, Aline Tecchio; TEO, Carla Rosane Paz Arruda; MUSSIO, Bruna Roniza. Educação alimentar e nutricional no ambiente escolar: uma revisão integrativa. Revista Ibero-Americana de Estudos em Educação, v. 11, n. 3, p. 1441-1460, 2016.

BRASIL, Ministério da Saúde (2015). Vigitel Brasil 2014 Saúde Suplementar: Vigilância de fatores de risco e proteção para doenças crônicas por inquérito telefônico. Agência Nacional de Saúde Suplementar, Brasília.

BRASIL. Ministério da Saúde. Doenças desencadeadas pela obesidade. Ministério da Saúde 2020. 
BRASIL. Ministério da Saúde. Brasileiros atingem maior índice de obesidades nos últimos 13 anos. Ministério da Saúde 2019.

BRASIL. Ministério da Saúde. Caderno do gestor do PSE. Brasília: Ministério da Saúde, 2015.

CAVALCANTI, Christiane Leite et al. Educação nutricional em creches no município de Patos-PB. One, GMC, Albuquerque HN, organizadores. Saúde e Meio Ambiente: os desafios da interdisciplinariedade nos ciclos da vida humana. Campina Grande: Instituto Bioeducação, p. 101-17, 2017.

CAVALCANTI, Patricia Barreto; LUCENA, Carla Mousinho Ferreira; LUCENA, Pablo Leonid Carneiro. Programa Saúde na Escola: interpelações sobre ações de educação e saúde no Brasil. Textos \& Contextos (Porto Alegre), v. 14, n. 2, p. 387-402, 2015.

CARVALHO, F. F. B. A saúde vai à escola: a promoção da saúde em práticas pedagógicas. Physis Revista de Saúde Coletiva, Rio de Janeiro, v. 25, n. 4, p. 1207-1227, 2015.

CECCATTO' ${ }^{1}$, Daiane et al. A influência da mídia no consumo alimentar infantil: uma revisão da literatura. CONSELHO EDITORIAL, p. 140, 2018.

CERVATO-MANCUSO, Ana Maria; VINCHA, Kellem Regina Rosendo; SANTIAGO, Débora Aparecida. Educação Alimentar e Nutricional como prática de intervenção: reflexão e possibilidades de fortalecimento. Physis: Revista de Saúde Coletiva, v. 26, p. 225-249, 2016.

COSTA, Midiã Ribeiro. A influência da mídia televisiva nas escolhas alimentares das crianças e na obesidade infantil: uma revisão da literatura. 2017.

COUTO, A. N.; KLEINPAUL, W. V.; BORFE, L. et al. 0 ambiente escolar e as ações de promoção da saúde. Cinergis, Santa Cruz do Sul, v. 17, n. 4 (Supl.1), p. 378-383, out./dez. 2016

DE ALMEIDA PEREIRA, Mariana et al. Desafios e reflexões na implantação de um programa de educação alimentar e nutricional (EAN) em indivíduos com excesso de peso. Revista Brasileira em Promoção da Saúde, v. 28, n. 2, p. 290-296, 2015.

DE CARVALHO, Fernanda Medeiros et al. A influencia da mídia na alimentação infantil. 2016.

DE SOUZA, Sandra Maura. Estratégias para prevenção e controle de carências nutricionais na infância em creche pactuada no Programa Saúde na Escola no município de Ipaba-MG. 2015. 
FARIAS, Isabelle Carolline Veríssimo de et al . Análise da Intersetorialidade no Programa Saúde na Escola. Rev. bras. educ. med., Rio de Janeiro, v. 40, n. 2, p. 261-267, June 2016.

FERREIRA, Paula Daianny Alves Araújo et al. Caracterização do comportamento alimentar e estado nutricional de adultos. Motricidade, v. 14, n. 1, p. 252-258, 2018.

FELBERG, Elisangela Ferreira Brito; PINHEIRO, Marlene Nogueira; BATISTA, Eraldo Carlos. Fatores psicológicos e sociais associados à desnutrição infantil: um estudo bibliográfico. Revista Opara, v. 6, n. 1, p. 32-48, 2018. (FELBERG; PINHEIRO; BATISTA, 2018)

FREITAS, P. P., Assunção, A. Á., Bassi, I. B. \& Lopes, A. C. S. (2016). Excesso de peso e ambiente de trabalho no setor público municipal. Revista de Nutrição, 29(4), 519-527. doi: 10.1590/1678-98652016000400007

GIACOMELLI, Simone de Castro et al . Comércio informal e formal de alimentos no âmbito escolar de um município da região central do Rio Grande do Sul. Braz. J. Food Technol. Campinas, v. 20, e2016136, 2017.

IBGE, 2016 Instituto Brasileiro de Geografia e Estatística. Pesquisa Nacional de Saúde do Escolar 2015 Rio de Janeiro: IBGE, 2016.

LINHARES, Francisca Michelli Medeiros et al. Obesidade infantil: influência dos pais sobre a alimentação e estilo de vida dos filhos. Temas em Saúde, v. 16, n. 2, p. 460-48, 2016.

MACHADO, M. F. A. S.; GUBERT, F. A.; MEYER, A. P. G. F. V. et al. Programa saúde na escola: estratégia promotora de saúde na atenção básica do Brasil. J. Hum. Growth Dev., São Paulo, v. 25, n. 3, p. 307 - 312, 2015.

MARTINS, Maria Salomé et al. Análise das ações intersetoriais no programa saúde na escola. Revista Brasileira de Educação e Saúde, v. 10, n. 1, p. 3239, 2020.

MELO, Karen Muniz et al. Influência do comportamento dos pais durante a refeição e no excesso de peso na infância. Escola Anna Nery, v. 21, n. 4, 2017.

MENDES, L. V. As consequências da desnutrição no desenvolvimento físico e mental infantil. Fundação Telefônica. Disponível em (2016).

MOLINA, MCB; CADE, N. V.; FARIAS JUNIOR, J. C. Atividade física e comportamento sedentário em crianças de duas regiões de Vitória, entre 2007 e 2011: uma análise comparativa. 2018. Dissertação de Mestrado. Universidade Federal do Espírito Santo. 
PIMENTA, T. A. M.; ROCHA, R.; MARCONDES, N. A. V. Políticas públicas de intervenção na obesidade infantil no Brasil: uma breve análise da política nacional de alimentação e nutrição e política nacional de promoção da saúde. Cient Ciênc Biol Saúde, v.17, n.2, p. 139-146, 2015.

PEREIRA, Paulo Almeida; LOPES, Liliana Correia. Obesidade infantil: estudo em crianças num ATL. Millenium-Journal of Education, Technologies, and Health, n. 42, p. 105-125, 2016.

PRADO, Bárbara Grassi et al. Ações de educação alimentar e nutricional para escolares: um relato de experiência. Demetra: alimentação, nutrição \& saúde, v. 11, n. 2, p. 369-382, 2016.

PRADO, Bárbara Grassi et al. Consumo de alimentos na cantina escolar entre adolescentes de uma escola estadual de Várzea Grande-MT. Seminário Transdisciplinar da Saúde, n. 04, 2018.

PRETO, Clara et al. Avaliação dos fatores associados a Desnutrição infantil na Guiné-Bissau. Acta Portuguesa de Nutrição, n. 12, p. 14-17, 2018.

REIS, Jéssyca Valadares Cavalcante dos. Educação Física como agente preventivo da obesidade infantil. 2017.

ROSSI, Camila Elizandra et al. Fatores associados ao consumo alimentar na escola e ao sobrepeso/obesidade de escolares de 7-10 anos de Santa Catarina, Brasil. Ciência \& Saúde Coletiva [online]. 2019, v. 24.

SA, Miriam Ribeiro Calheiros de. Promoção da saúde e ações intersetoriais: foco no Programa Saúde na Escola. Cad. Saúde Pública, Rio de Janeiro, v. 36, n. 3, e00001620, 2020.

SA, Miriam Ribeiro Calheiros de et al. De toda maneira tem que andar junto: ações intersetoriais entre saúde e educação para crianças vivendo com a síndrome congênita do vírus Zika. Cad. Saúde Pública, Rio de Janeiro, v. 35, n. 12, e 00233718, 2019.

SANTANA, Marília Karla Laurentino; OLIVEIRA CM, Clemente HA. Influência da publicidade de alimentos direcionada ao público infantil na formação de hábitos alimentares. Revista Uni-rn. Junho/dezembro de, v. 14, n. 1, p. 2, 2015.

SANTOS, Valdinei Oliveira dos et al. Ações em prol da alimentação saudável em cantinas escolares de Dourados-MS: impressões e atitudes dos gestores. 2018.

SILVA, André Luís Façanha da et al. Saúde e educação pelo trabalho: reflexões acerca do PET-Saúde como proposta de formação para o Sistema Único de Saúde. Interface - Comunicação, Saúde, Educação [online]. 2015, v. 19. 
SOUSA, Marta Caires de; ESPERIDIÃO, Monique Azevedo; MEDINA, Maria Guadalupe. A intersetorialidade no Programa Saúde na Escola: avaliação do processo político-gerencial e das práticas de trabalho. Ciência \& Saúde Coletiva, v. 22, p. 1781-1790, 2017.

TASCA, Paula Cristina et al. O SEDENTARISMO E A OBESIDADE INFANTIL NA CONTEMPORANEIDADE. Seminário de Iniciação Científica, Seminário Integrado de Ensino, Pesquisa e Extensão e Mostra Universitária, 2018.

TORAL, N., Gubert, M. B., Spaniol, A. M. \& Monteiro, R. A. (2016). Eating disorders and body image satisfaction among Brazilian undergraduate nutrition students and dietitians. Archivo Latinoamericano de Nuticion, 66(2), 129-134

VIANA, Teresinha Cícera Teodoro et al. Fatores determinantes da desnutrição infantil em crianças de 0 a 5 anos cadastradas no programa de crescimento e desenvolvimento infantil na Amazônia Legal. 2018. 